\title{
Hardness of modular factorial
}

\author{
Thinh D. NGUYen* \\ Moscow State University \\ kosmofarmer@gmail.com
}

July 29, 2018

\begin{abstract}
In this note, we show that computing modular factorial (MODFACT) is a very hard task.
\end{abstract}

\section{Definition}

We give the definition of what is the modular factorial function problem.

\section{Definition:}

ModFACT: Given $n, m$ such that $m \leq n$, compute $(m ! \bmod n)$

In the following sections, we will give the application of MoDFACT in some branches of mathematics - in particular number theory.

\section{Primality testing}

This section only defends the following observation.

OBSERVATION

If one can compute MoDFACT, one can easily do primality testing. Indeed, by using Wilson's theorem, primality testing is a direct application of MODFACT.

WiLSON'S THEOREM: For $p \geq 2$, we have $(p-1) ! \equiv-1 \bmod p$

\footnotetext{
*Perebor
} 
Proof: Let $p$ be a prime; the claim is trivial if $p=2$, so assume that $p$ is odd. The idea is to look at pairs of the elements of $(\mathbb{Z} / p \mathbb{Z})^{\times}$. In fact, for every $a \in(\mathbb{Z} / p \mathbb{Z})^{\times}$there is an element $a^{-1} \in(\mathbb{Z} / p \mathbb{Z})^{\times}$such that $a \cdot a^{-1} \equiv 1 \bmod p$. In general, $[a]$ and $\left[a^{-1}\right]$ are distinct: $[a]=\left[a^{-1}\right]$ implies $\left[a^{2}\right]=[1]$, so this can only happen (and does in fact happen) if $[a]=[1]$ or $[a]=[-1]=[p-1]$ (here we use that $(\mathbb{Z} / p \mathbb{Z})$ is a field; in fields, polynomials of degree 2 such as $x^{2}-1$ have at most 2 roots).

Thus, $(\mathbb{Z} / p \mathbb{Z})^{\times} \backslash\{[-1],[+1]\}$ is the union of pairs $\left\{[a],\left[a^{-1}\right]\right\}$ with $[a] \neq$ $\left[a^{-1}\right]$, hence the product over all elements of $(\mathbb{Z} / p \mathbb{Z})^{\times} \backslash\{[-1],[+1]\}$ must be [1]. We can get $[(p-1)$ !] by multiplying this product with the two missing classes $[1]$ and $[-1]$, and this gives the claimed result $[(p-1) !]=[-1]$.

We still have to prove the converse: assume that $(n-1) ! \equiv-1 \bmod n$; if $p$ is a prime divisor of $n$, this congruence implies $(n-1) ! \equiv-1 \bmod p$. But $p \leq n-1$ also implies that $p$ occurs as factor of $(n-1)$ ! on the left hand side, hence we would have $0 \equiv(n-1) ! \bmod p$. But then $0 \equiv-1 \bmod p$, a contradiction.Q.E.D

\section{SQUARE ROOTS OF -1}

First, we give the following observation.

\section{OBSERVATION}

Let $p$ be an odd prime and put $a=\left(\frac{p-1}{2}\right) ! ;$ then we have that $a^{2} \equiv$ $(-1)^{(p-1) / 2} \bmod p$. In particular, $a \equiv \pm 1 \bmod p$ if $p \equiv 3 \bmod 4$, and $a^{2} \equiv$ $-1 \bmod p$ if $p \equiv 1 \bmod 4$.

Proof: We start with Wilson's theorem $(p-1) ! \equiv-1 \bmod p$; if, in the product $(p-1)$ !, we replace the elements $\frac{p+1}{2}, \frac{p+3}{2}, \ldots, p-1$ by their negatives $-\frac{p+1}{2} \equiv \frac{p-1}{2},-\frac{p+3}{2} \equiv \frac{p-3}{2}, \ldots,-(p-1) \equiv 1 \bmod p$, then we have introduced exactly $\frac{p-1}{2}$ factors -1 ; thus $(p-1) ! \equiv(-1)^{(p-1) / 2} a^{2} \bmod p$ with $a=\left(\frac{p-1}{2}\right)$ !. This proves the claim.Q.E.D

Using this observations, it is fairly straightforward to compute a square root of -1 modulo a prime $p$ congruent to 3 modulo 4 . But in the opposite direction, if we somehow compute both square roots (if one can compute one, then he can compute both) of -1 efficiently, we do not know which one among the two returned values is the modular factorial $a=\left(\frac{p-1}{2}\right) ! \bmod p$. In a sense, computing square roots of -1 is easier than computing modular factorial. 


\section{SUM OF TWO SQUARES}

The following claim, together with the previous observation, will be necessary tools to shine lights on the observation of this section.

Claim: Given an integer a not divisible by $p$, there exists $x, y \in \mathbb{Z}$ with $1 \leq|x|,|y| \leq \sqrt{p}-1$ such that ay $\equiv x \bmod p$.

ProOF:Let $f$ be the smallest integer greater than $\sqrt{p}$, and consider the residue classes $\{[u+a v]: 0 \leq u, v \leq f-1\}$ modulo $p$. There are $f^{2} \geq p+1$ such expressions, but only $p$ different residue classes, hence there must exist $u, u^{\prime}, v, v^{\prime}$ such that $u+a v \equiv u^{\prime}+a v^{\prime} \bmod p$. Put $x=u-u^{\prime}$ and $y=v-v^{\prime}$; then $x \equiv a y \bmod p$, and moreover $-f<x, y<f$.Q.E.D

Now, we are ready to give the observation of this section. OBSERVATION

Every prime $p \equiv 1 \bmod 4$ is a sum of two integral squares.

Proof: Since $p \equiv 1 \bmod 4$, there is an $a \in \mathbb{Z}$ such that $a^{2}=-1 \bmod p$. Using the previous claim, we imply that there are integers $x$ and $y$ such that $a y \equiv x \bmod p$ and $0<x, y<\sqrt{p}$. Squaring gives $-y^{2} \equiv x^{2} \bmod p$, that is $x^{2}+y^{2} \equiv 0 \bmod p$. Since $0<x^{2}, y^{2}<p$, we find that $0<x^{2}+y^{2}<2 p$; since $x^{2}+y^{2}$ is divisible by $p$, we must have $x^{2}+y^{2}=p$.Q.E.D

That the solution to $p=x^{2}+y^{2}$ in this case is unique is also known since Gauss. It is also worth noting that computing modular factorial does not help much to find a representation of a prime as sum of two squares. But in this case, another hard problem, namely USAT, can really of help.

\section{DEFINITION}

$\mathrm{USAT}=\{\Phi-$ the Boolean formula $\Phi$ has exactly one satisfying variable assignment\}

Clearly, given the uniqueness of solution to $p=x^{2}+y^{2}$ when $p \equiv 1 \bmod 4$, the problem of finding such a representation is reducible to USAT. So, we see here that hardness of problems does not come alone but comes in many.

\section{REFERENCES}

[1] Michael R. Garey, David S. Johnson, Computers and Intractability: A Guide to the Theory of NP-Completeness 
[2] Phan Dinh Dieu, Le Cong Thanh, Le Tuan Hoa, Average Polynomial Time Complexity of Some NP-Complete Problems. Theor. Comput. Sci. 46(3): 219-237 (1986)

[3] L. J. Mordell, Diophantine equations, St. John's College, Cambridge, England, 1969

[4] Franz Lemmermeyer, Elementary Number Theory - Chap. 5, http://www.fen.bilkent.edu.tr/ franz/nt/ch5.pdf 